\title{
“Space Worm": Bore-Hole Anchoring Mechanism for Micro-G Planetary Exploration Drill
}

\author{
Hugo Gagnon ${ }^{*}$, Emile Abou-Khalil", Omar Azrak ${ }^{*}$, Alexei Morozov ${ }^{*}$, Howard Jones ${ }^{* *}$, \\ and Gita Ravindran** \\ *Department of Mechanical Engineering, McGill University, Montreal, Quebec, Canada \\ **MDA Corporation, Brampton, Ontario, Canada \\ hugo.gagnon2@mail.mcgill.ca, emile.abou-khalil@mail.mcgill.ca, \\ mohammad.azrak@mail.mcgill.ca, alexei.morozov@mcgill.ca, \\ howard.jones@mdacorporation.com and gita.ravindran@mdacorporation.com
}

\begin{abstract}
Planetary drilling has become an essential need in the search for life or resource identification on the Moon and other near-Earth objects. This paper focuses on the mechanical design aspects of $a$ bore-hole anchoring mechanism architecture that is relatively independent from its "mother" vehicle. The recommended solution comprises a two member actuated anchoring mechanism. Each actuator is driven by one leading screw thus providing a considerable mechanical advantage to the anchor shoes. The anchor's ability to "walk" up and down the hole is similar to that of an earthworm, hence the naming "Space Worm". The present paper is not an extensive technical solution to a planetary drilling unit, but rather proposes general concepts that could eventually constitute the backbone of such a sophisticated machine.
\end{abstract}

\section{Introduction}

As humanity has become more and more technologically advanced since the first man landed onto the Moon, new frontiers have been pushed towards space exploration. Nowadays, planetary drilling applications such as paleoclimatology, geology and the search for life or resource identification on Mars require newly developed techniques. MDA Corporation [1] is one amongst the engineering force whose role is to spark and support inventions that will eventually meet the difficult design objectives that are often associated with space constraints, such as light components and tight dimensions. The project described here is the continuity of a large scale effort in finding planetary drilling solutions inspired by MDA Corporation. Previous papers related to this one include descriptions of a drill rod interface and auxiliary mechanism [2] as well as a drill bit and sample acquisition mechanism [3]. All of these projects, including this one, were part of a final undergraduate course in Mechanical Engineering of McGill University.

Pre-existing drilling mechanisms used to retrieve regolith samples require the use of numerous inter-connected rods that can be pushed against each other from the Earth's surface to provide thrust to the drill bit located down-hole[4]. However, this technique is very costly and it is cumbersome to send heavy anchoring and drilling mechanisms on planetary missions. Furthermore, this design has inherent limits to the achievable depth due to mass and power limitations. Hence, it is of interest to find a new method to retrieve samples; one that would eliminate the need for using a multiple rod system. Such a device should have the ability to 'walk' down and then up a hole without being pushed in any way by any other means than its own mechanical components. Advantages resulting from such a concept are obvious. For example, the assembly could be allowed to drill as much as it is deemed necessary, instead of being constrained to a certain depth due to the lack of extra rods.

\section{Design Layout}

The Space Worm (Fig. 1) is composed of a top and bottom shell portion, with each one of the two housing an anchoring mechanism. The shells are transparent in this prototype to help visualize 
the inside mechanisms. The bottom shell can slide partially inside the top one. The shells are a quasi mirror image of each other from the horizontal plane taken at the middle of the assembly. This symmetry, among other things, is the reason why the Space Worm can "walk" up and down a hole interchangeably. However, the most innovative part of the Space Worm is that it makes use of only two actuators, or motors, to provide both the necessary anchoring and the thrust generating forces. Further details on these topics will be discussed extensively in subsequent sections.

The drill bit interface must be attached to the lower part of the Space Worm. The upper part would then be linked to a power transmitting rover by means of a loose tether.

The exploded view of the Space Worm is shown in Fig. 2. All components are listed in Table 1. To facilitate assembly, both the top and bottom shells are cut vertically into two halves. For each shell, there is one end (1) and two stoppers (4) that have two holes each located 180 degrees apart on their sides while the motor holder (3) has two holes on only one side. Using simple screws, these holes permit the corresponding two half shells $(10,11$ or 12,13$)$ to be clamped around the above mentioned inner components. There are two kinds of top and bottom half shells because only one of the top and bottom halves has holes to accommodate the motor holder. This particularity of the motor holder is due to space constraints. The ends, which are not shown on Fig.1, are meant to seal the assembly from debris intrusion while at the same time reinforcing the shell clamping force.

The Space Worm's core mechanism is in reality two subassembly sets of inter-connecting components. Both sets are identical and there is one set associated to each shell. For each shell, a motor holder (3) is fixed on it. The motor holder holds a motor (2). The motor shaft is fixed to a leading screw (9). The leading screw is connected to a nut (14) located in the opposing shell of the motor-leading screw connection. For instance, the motor in the top shell is connected via a leading screw to a nut located in the bottom shell and vice versa. The nut is embedded inside a holder (5) so that, from now on, the nut and the holder can be considered as one piece. The holder is connected to two sets of two arms (6). Each set of arms links the holder with one shoe

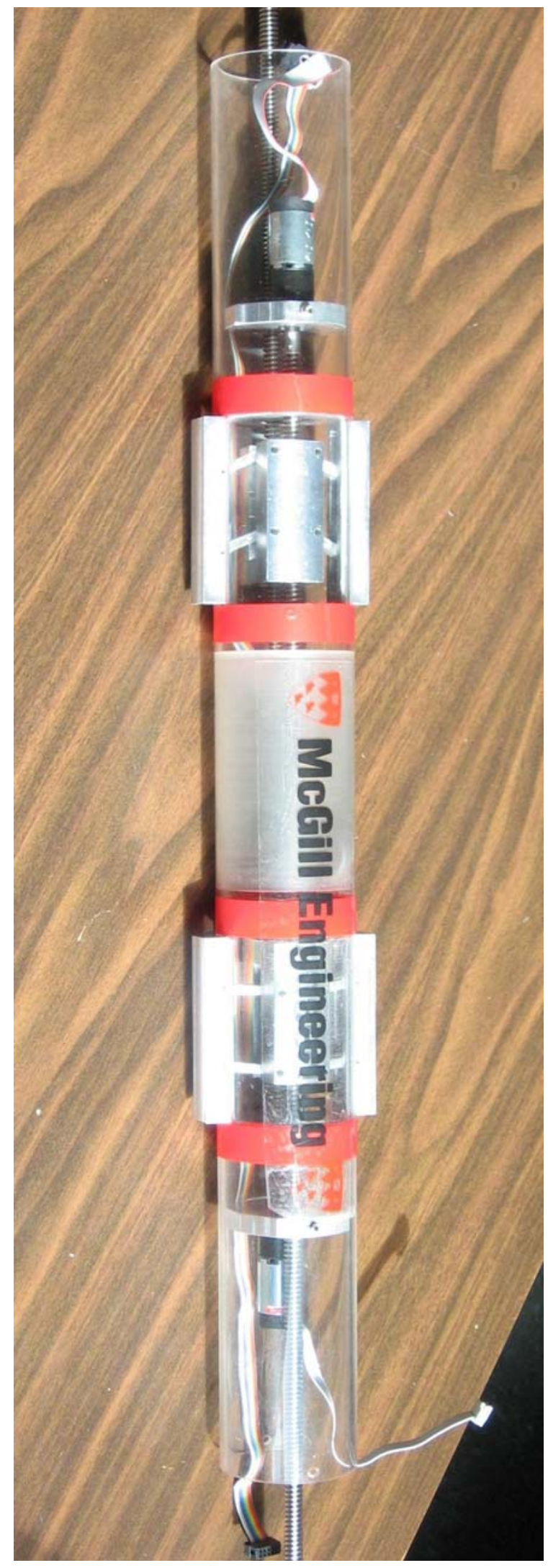

Figure 1. Space Worm 


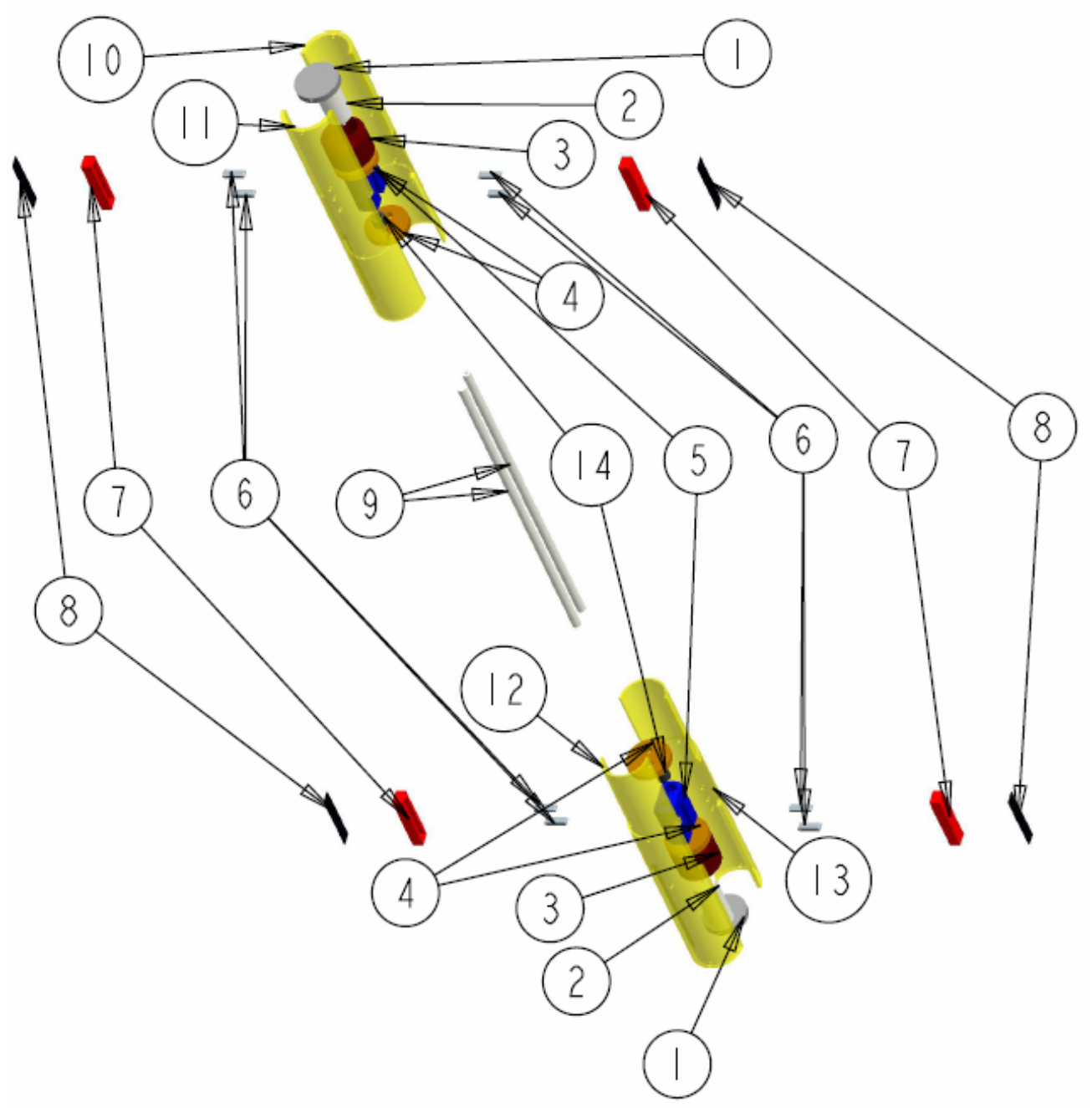

Figure 2. Exploded assembly

Table 1. Part names and quantities

\begin{tabular}{|c|c|c|}
\hline$\#$ & Part Name & Quantity \\
\hline $\mathbf{1}$ & End & 2 \\
\hline $\mathbf{2}$ & Motor & 2 \\
\hline $\mathbf{3}$ & Motor Holder & 2 \\
\hline $\mathbf{4}$ & Stopper & 4 \\
\hline $\mathbf{5}$ & Holder & 2 \\
\hline $\mathbf{6}$ & Arm & 8 \\
\hline $\mathbf{7}$ & Shoe & 4 \\
\hline $\mathbf{8}$ & Shoe Tip & 4 \\
\hline $\mathbf{9}$ & Leading Screw & 2 \\
\hline $\mathbf{1 0}$ & Top Shell 1 & 1 \\
\hline $\mathbf{1 1}$ & Top Shell 2 & 1 \\
\hline $\mathbf{1 2}$ & Bottom Shell 1 & 1 \\
\hline $\mathbf{1 3}$ & Bottom Shell 2 & 1 \\
\hline $\mathbf{1 4}$ & Nut & 2 \\
\hline
\end{tabular}


(7). Finally, to allow for a higher coefficient of friction between the shoes and the bore-hole walls, shoe tips (8) are glued onto the anchor shoes.

Now that the design terminology has been laid out, the main concepts underlying the Space Worm will be explored in detail.

\section{Concept Explanation}

The functioning of the Space Worm rests upon a complex set of forces and reactions. Before going into any further detail, it is worth recalling the notions associated with a leading screw and a nut [5]. When a nut is held firmly onto a leading screw and the leading screw is rotating, then by action-reaction the leading screw will either move upward or downward depending on its rotational direction. Inversely, if a leading screw is fixed while a nut is rotating, then the nut will translate along the leading screw.

From the very beginning of the concept generation phase, the problem statement was divided into two main categories: the anchoring and the actuating or thrust generating mechanisms. The final selected concept for each one of the two categories is explained below.

\subsection{Anchoring Mechanism}

The anchoring mechanism's function is selfexplanatory; it anchors or prevents slippage between the anchor shoes and the bore-hole walls due to either gravity or to the countering drilling force.

The concept is illustrated in Fig. 3. Each anchor shoe is constrained horizontally and connected by pin joints to its respective set of two arms. The arms are also connected by pin joints to a holder. Therefore, in order to retract or expand the shoes from the shell, the holder has to move either downward or upward. Since a nut is embedded inside the holder, the holder follows the nut's motion which is itself dependent on the leading screw rotation. Since the arms are attached to the holders and shoes by pin joints, the arms rotate according to the holder's vertical translation and the shoes' horizontal translations.

Note that each shoe is linked to a set of two arms. The reason is to constrain the shoe's movement in a pure horizontal translation instead of also involving rotational aspects arising from assembly misalignments. Rotation of the shoes is not desired as it would restrain the anchoring mechanism from expanding.

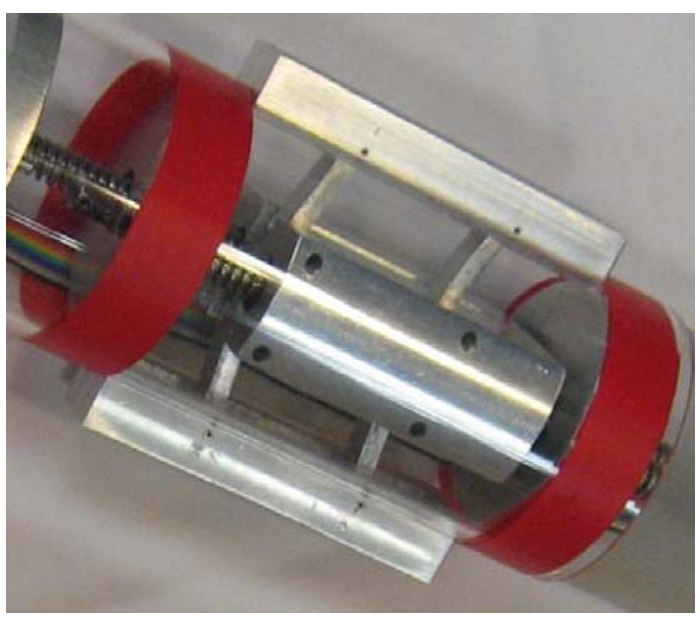

Figure 3. Anchoring mechanism

\subsection{Thrust Generating Mechanism}

The thrust or actuating mechanism is responsible for providing weight on the drill bit during drilling. Although the functioning of the Space Worm was decomposed in two separate categories to facilitate understanding, it will now be shown that the functioning of the thrust generating mechanism actually derives from the continuity of the anchoring mechanism motion. As will be pointed out in subsequent sections, this feature allows the Space Worm to use only two motors instead of three or more to accomplish the same tasks.

The shoes can deploy or retract until a certain limit has been reached. This limit comes when the holder corresponding to the shoes hits a boundary. In real time functioning, this boundary can either be physical or artificial. The physical boundary refers to the physical contact between the holder and one of its two corresponding stoppers. Fig. 4 illustrates this specific boundary state. On the other hand, the artificial boundary refers to the shoes touching the bore-hole walls before their holder reaches a stopper. For the sake of concept comprehensiveness, the physical boundary state will be assumed for the following demonstration. Note that whether it be for a physical or artificial boundary state, the shoes stop moving when the holder reaches one of the two. 

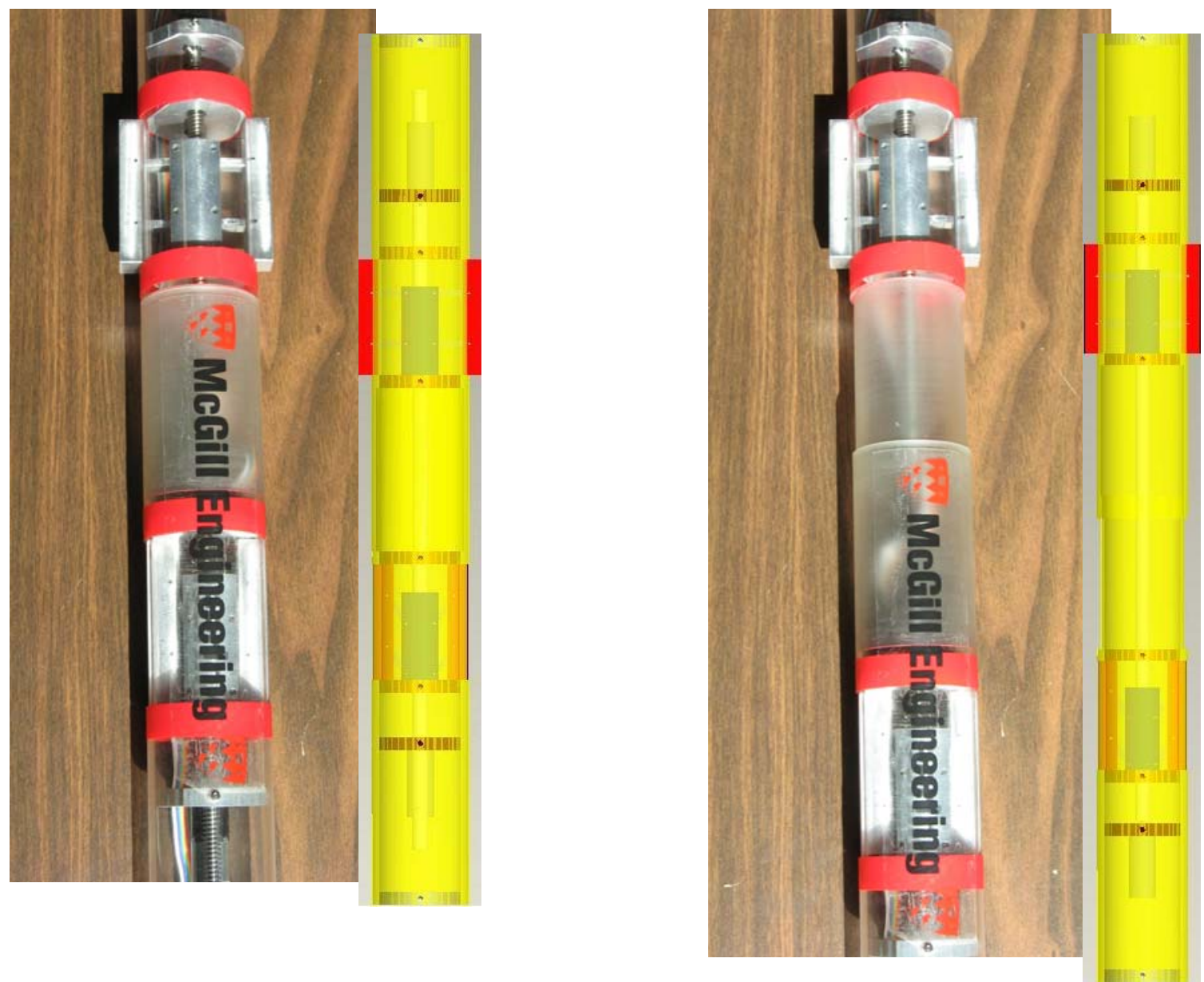

Figure 4. Thrust generating mechanism left: retracted, right: expanded

The actuating mechanism can either retract or expand the shells relative to one another. Shell expansion is first described. Recall that the holder and therefore the nut located in the bottom shell is connected to the motor via a leading screw located in the top shell, and vice versa. Looking at the left drawing of Fig. 4, if the top motor continues to rotate once the bottom holder reaches the bottom stopper of the bottom anchoring mechanism, then this holder will start pushing against that particular stopper. Assuming the top motor to be stationary and neglecting the top anchoring mechanism for now, the resulting consequence would be the bottom shell moving downward. Now, since the top motor is fixed on the top shell by a motor holder, the assumption of taking the top motor to be stationary is in reality anchoring the top shoes against the bore-hole walls (as illustrated in Fig. 4). Thus, considering the top anchoring mechanism in this analysis, one has to visualize that the bottom motor has to rotate simultaneously compared to the top one in order to keep the top anchoring mechanism expanded or anchored as the bottom shell moves downward. If this requirement is not satisfied, or one motor runs faster than the other one, then, depending on the relative rotational speed of the motors, either large stresses will be induced or the top anchoring mechanism will be retracted. If the latter case occurs, then nothing will be maintaining the top shell stationary and the application of a drilling force will no longer be feasible. The right drawing in Fig. 4 shows the Space Worm when fully expanded, that is when each nut reaches the extremity of their corresponding leading screw.

In terms of boundary states for this particular stroke, although the top anchoring mechanism refers to a physical boundary on Fig. 4, in practice it should involve an artificial boundary 
as the shoes would be partially expanded onto the bore-hole walls whereas the bottom anchoring mechanism is, as illustrated, associated with a physical boundary as the holder pushes against the stopper to provide thrust. Note that shell expansion of the top shell in the upward direction is also possible by virtue of symmetry.

In essence, the underlying concepts of shell retraction are similar. It is now assumed that the Space Worm's initial position is the same as the one illustrated by the right drawing of Fig. 4. Instead of rotating the top motor in order to push the bottom holder downward as was the case previously, the bottom motor is now rotated so that the top holder starts pressing against the bottom stopper of the top anchoring mechanism. Note that in reality, the top holder does not reach the stopper since an artificial boundary state is involved. In any of the two cases, the result remains the same: since the top anchoring mechanism is already anchored, the bottom shell will start moving upward until it reaches the position shown on the left drawing of Fig. 4. However, as in the previous case, the top motor has to rotate simultaneously compared to the bottom one to maintain the bottom anchoring mechanism retracted or unanchored while the bottom shell moves upward. Note that retraction of the top shell in the downward direction is also possible by virtue of symmetry.

It is now apparent that both the anchoring and thrust generating mechanisms capabilities result from the same linear actuators, as opposed to, for example, using one actuator for each anchoring mechanism and a third one to provide thrust.

Benefits resulting from a two member actuated anchoring mechanism are numerous. For instance, it considerably diminishes the complexity of the whole system and may reduce energy consumption. The energy issue is a significant one as the rover has inherent limits to the number of tasks it can accomplish for a given period of time on Mars. However, this gain in saving one motor has also its disadvantages. For example, expensive and quite complex controls are required to properly start and stop both motors simultaneously during shell expansion and retraction.

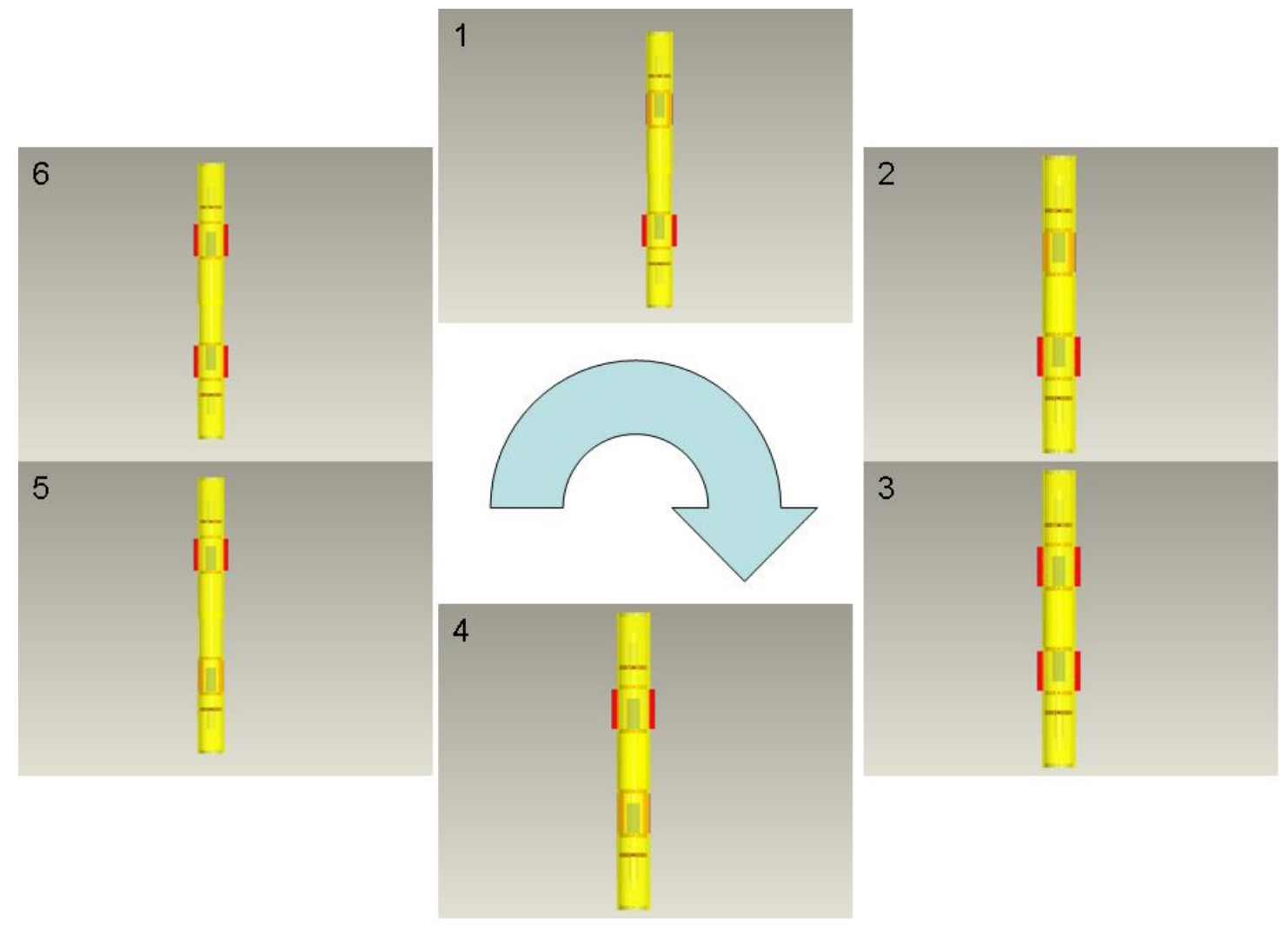

Figure 5. Six-step cycle 
The concepts discussed above can now be used to construct one full cycle that the Space Worm has to perform in order to achieve greater depths.

\section{Cycling}

As seen from Fig. 5, it requires six fully independent steps in order to achieve one cycle. Positions (4) to (5) are the only steps which give the drill bit the necessary force to drill further down the hole, thus permitting regolith sample acquisitions. This special step is called the power stroke. All other steps are meant to prepare the Space Worm for the next power stroke. The steps should be followed sequentially. Note that the shells are retracted from positions (2) to (4) and expanded from positions (5) to (1).

Each step is briefly discussed next. For each step, the general motions of the shells are stated, followed by a brief description of the motor behaviours. The underlying concepts behind the anchoring and thrust generating mechanisms described in section 3 are assumed to be understood.

The two subassemblies, each constituted by a motor and its corresponding anchoring mechanism, are illustrated for the six positions (Fig. 6). These positions are meant to illustrate two features: first, the relative displacements of the holders as the shells are being expanded or retracted and second, the shoes' expansion or retraction within the shells.

\section{(1) to (2) - Upper shell retracted to bottom shell}

The top motor rotates until the top shell is fully retracted. Simultaneously, the bottom motor rotates to keep the top shoes retracted.

(2) to (3) - Top anchors deployed, bottom anchors stationary

The top motor is locked. Meanwhile, the bottom motor rotates until the top shoes are fully deployed.

(3) to (4) - Top anchors stationary, bottom anchors retracted

The top motor rotates until the bottom shoes are fully retracted. Meanwhile, the bottom motor is locked.
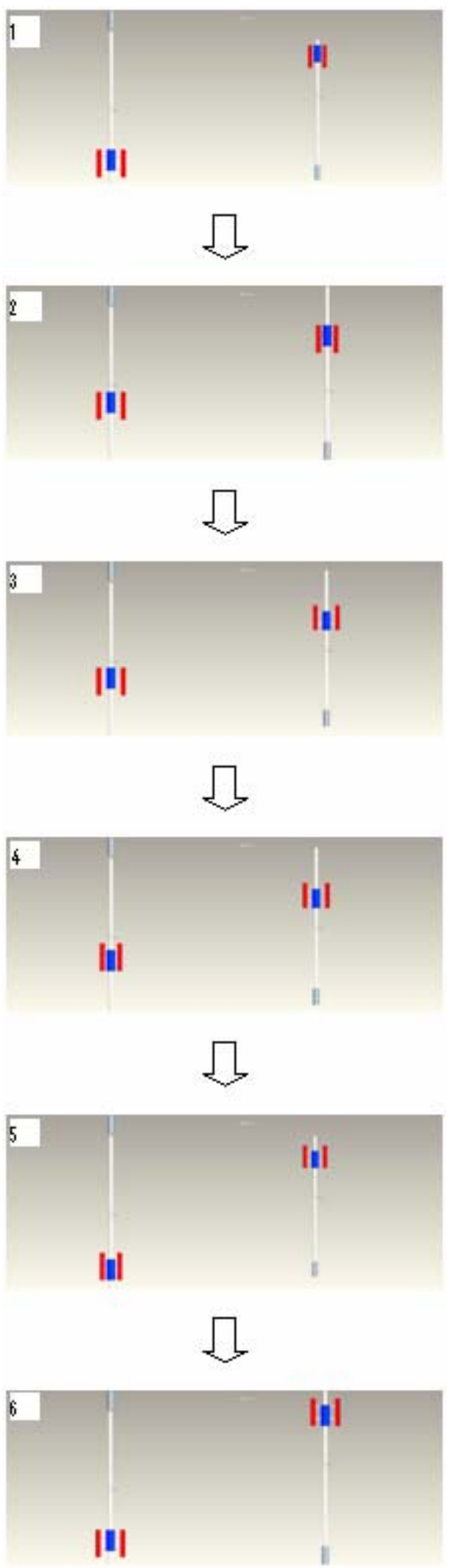

Figure 6. Top and bottom subassemblies relative positions during cycle 
(4) to (5) - Bottom shell pushed downward (Power Stroke)

The top motor rotates until bottom shell is fully expanded. Simultaneously, the bottom motor rotates to keep the top shoes deployed.

(5) to (6) - Top anchors stationary, bottom anchors deployed

The top motor rotates until the bottom shoes are fully deployed. Meanwhile, the bottom motor is locked.

(6) to (1) - Top anchors retracted, bottom anchors stationary

And finally, the top motor is locked. Meanwhile, the bottom motor rotates until the top shoes are fully retracted.

Again, note that positions (1) to (2) and (4) to (5) require both motors to rotate simultaneously and at the exact same speed since while the shells are expanding or retracting relative to each other, only one of the two anchoring mechanism must be anchored. Otherwise, if both anchors are deployed at the same time during the shell's expansion or retraction, large amount of stresses will be induced in the prototype which in turn may result in a catastrophic failure.

Nature has always been a source of inspiration to humans and in many cases the resulting inventions have proven to be adequate and of great interest to Mankind [6]. Notice the resemblance between the Space Worm and an earthworm. Similar to an earthworm, the Space Worm grinds the soil then forces the particles along its body towards its rear. Moreover, the Space Worm progresses much like an earthworm by elongating and then shortening its overall length, and also by applying the necessary pressure on bore-hole walls to allow displacement.

\section{Analysis}

A brief analysis of general internal forces is shown in the following section. The purpose of this study is to highlight the mechanical advantages pertaining to the Space Worm and the impact of using a two motor configuration in terms of required controls.

\subsection{Mechanical Advantage}

The use of a leading screw in the role of a linear actuator provides a considerable mechanical advantage to the motors as opposed to, for example, linear actuators driven by electromagnetic forces [7]. Large mechanical advantage values are of course of great interest because smaller motors can be used while still providing the necessary driving torque. Since smaller motors allow for a smaller scaling of the downhole assembly, the overall resulting assembly also becomes lighter and thus cheaper to deploy on planetary missions. In order to select the proper motor, a force analysis must be derived. A quick glance at the forces involved at the shoe tips of the Space Worm is given next. The free body diagram of an anchored shoe is illustrated in Fig. 7.

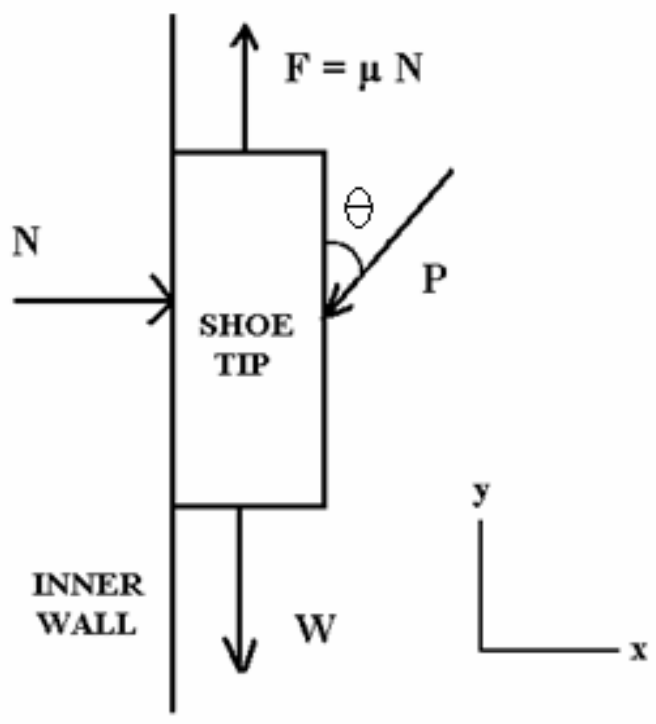

Figure 7. Free body diagram of shoe tip

Summing all forces in $\mathrm{X}$ and $\mathrm{Y}$ direction and setting acceleration to zero, the resulting equations are:

in X-direction

$$
N=P \sin (\theta)
$$

in Y-direction

$$
W=F-P \sin (\theta)
$$


Taking $F=\mu N$ and combining the equations in $\mathrm{X}$ and $\mathrm{Y}$ in matrix form gives the following

$$
\left[\begin{array}{rr}
1 & -\sin (\theta) \\
\mu & -\sin (\theta)
\end{array}\right]\left[\begin{array}{l}
N \\
P
\end{array}\right]=\left[\begin{array}{c}
0 \\
W
\end{array}\right]
$$

Solving this linear system of two equations and two unknowns yields the forces $\mathrm{N}$ and $\mathrm{P}$. The driving torque $T_{d}$ can be found by inserting $P$ in the equation included in fig. 8, where $\mathrm{L}$ is the screw lead and e is the screw efficiency. The screw lead is the distance travelled by a nut per turn. The screw efficiency varies from $50 \%$ for a standard ACME lead screw to $90 \%$ for a ball screw [8]. One important observation is to notice how the driving torque varies corresponding to the screw lead. As L gets smaller, the higher the mechanical advantage becomes and therefore a lower driving torque is required to provide the same thrust to the drill bit.

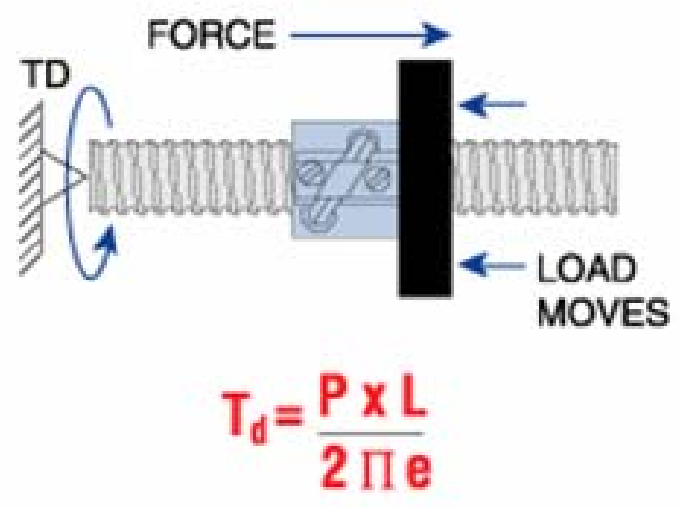

Figure 8. Driving torque formula

Another significant mechanical advantage can be found in selecting a proper planetary gear head when purchasing the motors [9]. Since planetary drilling is normally not constrained by time, large gear heads can be chosen even though they reduce the rotational speed of the motor shaft. Specifically, in the course of this year's mechanical engineering project, a gear ratio of 84:1 was used. Adding up the two mechanical advantages allow for a wider range of motor selection. Moreover, since gravity is not as intense on Mars as it is on Earth, the motors can be chosen accordingly. In fact, a micro-G environment eases the climbing up or ascending phase of the Space Worm.

\subsection{Controls}

The fact that both motors have to rotate simultaneously is the very reason why the controls needed are indeed somewhat specific and very costly. Software needs to be implemented so that all six steps of the cycle run smoothly. A proposed configuration fulfilling such requirements consists an electronic positioning system (EPOS) connecting the motors, the power supply and the user interface. The EPOS can be monitored using easily accessible software such as LabVIEW, or even with programming languages such as Fortran, $\mathrm{C}++$ and Visual Basics [9].

\section{Recommendations}

Since this paper only focuses on a proof of concept, it goes without saying that the actual design of the Space Worm should be modified considerably to meet the strict requirements associated with planetary drilling. Two major modifications are proposed here. They involve shoe anchoring and debris protection.

A better grip on the bore-hole walls could be achieved by modifying the size and number of shoes of the anchoring mechanisms. For the scope of this paper, only two shoes were incorporated in the anchoring mechanisms, but the holder could also be designed to house three or more sets of arms. This possibility, combined with better shoe tip materials, would definitely make more efficient us of the bore-hole circumference. Because the Space Worm should be able to anchor over a wide range of wall integrity, the shoe tip texture should be chosen according to the inner wall material, such as loosely consolidated regolith.

The Space Worm was designed as to minimize debris intrusion within the internal mechanisms. However, due to thermal variations related to planetary drilling, it anticipated that a planetary exploration drill system may experience variance in its dimensions. It is thus essential to obstruct the potential openings such as the space around the shoes or the shell's sliding line. It is recommended that O-rings be installed at those locations. Moreover, to avoid the shell expansion or retraction phases to jam due to large nongrinded particles, it is also recommended to shape the shells appropriately. As an example, Fig. 9 illustrates the possibility of incorporating 
grooves to facilitate dirt channelling as the Space Worm progresses down hole.

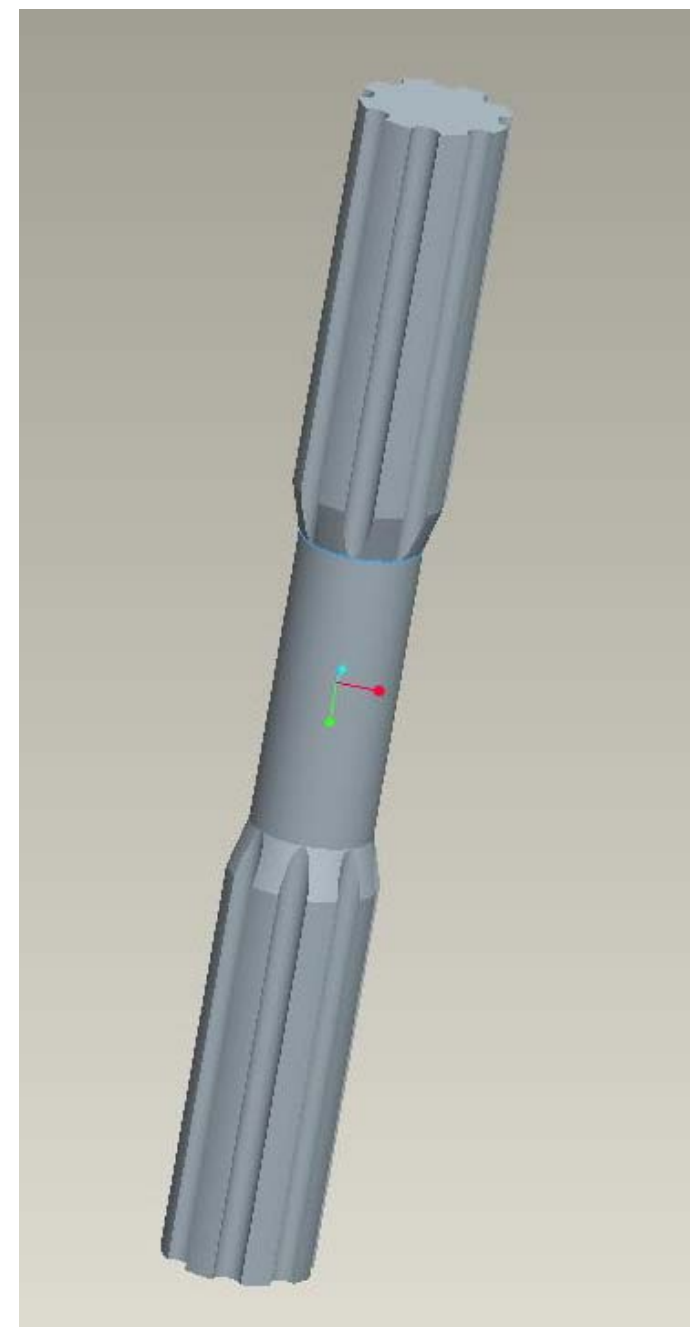

Figure 9. Indented grooves in shells

\section{Conclusion}

This paper demonstrates the design possibility of a down-hole anchoring assembly using two linear actuators. Benefits to symmetry include the Space Worm's capability to "walk" up and down a hole interchangeably. The most innovative feature is however the completion of a full cycle using only two motors while still exploiting the mechanical advantage of leading screws. Many advantages result from such a gain in space and power, including economical savings. Disadvantages include the need for more specific and expansive controls. Some recommendations were pointed out to take into account realities associated with planetary drilling such as debris intrusion.

\section{References}

[1] MDA Corporation, http://www.mdacorporation.com/, 2007.

[2] Marcil, J., Sabbag, P., Nolet, J-P., Jones, H., Morozov, A., "The Design of Drill Rod Interfaces and Their Auxiliary Mechanism: DRIAM”, Proc. The $2^{\text {nd }}$ International CDEN Design Conference, Kananaskis, Canada, July 17-20 2005.

[3] Bhaiji, M., El-Saddi, M., El-Khouri, N., Jones, H., Morozov, A., "The Design of the Drill Bit \& Sample Acquisition Mechanism: DBSAM”, Proc. The $2^{\text {nd }}$ International CDEN Design Conference, Kananaskis, Canada, July 17-20 2005.

[4] Ketchum, Milo S., The design of mine structures, New York: McGram-Hill, 1992.

[5] Edited by Manjriker Gunaratne, The foundation engineering handbook, FL : CRC/Taylor \& Francis, 2006.

[6] Edited by Yoseph Bar-Cohen , Biomimetics : biologically inspired technologies, FL : CRC/Taylor \& Francis, 2006.

[7] Boldea, I., Linear electric actuators and generators, New York: Cambridge University Press, 1997.

[8] Shigley, Joseph E., Mischke, Charles R., Budynas, Richard G., Mechanical engineering design, 2003.

[9] Maxxon Motors, http://www.maxonmotor.com/, 2007.

[10] Electromate Industrial Sales, http://www.electromate.com/, 2007. 